3-12-2019

\title{
Bayesian Approximation Techniques for Scale Parameter of Laplace Distribution
}

Uzma Jan

University of Kashmir, Srinagar, peeruzma1@gmail.com

S.P.Ahmad

University of Kashmir, Srinagar, sprvz@yahoo.com

Follow this and additional works at: https://digitalcommons.wayne.edu/jmasm

Part of the Applied Statistics Commons, Social and Behavioral Sciences Commons, and the Statistical Theory Commons

\section{Recommended Citation}

Jan, U., \& Ahmad, S. P. (2018). Bayesian approximation techniques for scale parameter of Laplace distribution. Journal of Modern Applied Statistical Methods, 17(2), eP2625. doi: 10.22237/jmasm/1552409925

This Regular Article is brought to you for free and open access by the Open Access Journals at DigitalCommons@WayneState. It has been accepted for inclusion in Journal of Modern Applied Statistical Methods by an authorized editor of DigitalCommons@WayneState. 


\section{Bayesian Approximation Techniques for Scale Parameter of Laplace Distribution}

\section{Erratum}

In the original published version of this article, the caption for Table 4 was incorrectly given as "Posterior estimates of Laplace distribution using normal approximation" instead of "Posterior estimates of Laplace distribution using T-K approximation". This has been corrected. 


\section{Bayesian Approximation Techniques for Scale Parameter of Laplace Distribution}

\section{Uzma Jan}

University of Kashmir

Srinagar, India

\author{
S. P. Ahmad \\ University of Kashmir \\ Srinagar, India
}

The Bayesian estimation of the scale parameter of a Laplace Distribution is obtained using two approximation techniques, like Normal approximation and Tierney and Kadane (T-K) approximation, under different informative priors.

Keywords: Bayesian estimation, prior distribution, normal approximation, $\mathrm{T}-\mathrm{K}$ approximation

\section{Introduction}

The Laplace distribution is a continuous probability distribution named after Pierre Simon Laplace (1749-1827) who, in 1774, obtained it as the distribution whose likelihood is maximized when the location parameter is set to the median. It is also known as the law of the difference between two independent variables with identical exponential distributions (Abramowitz \& Stegun, 1972), as the double exponential distribution because it can be thought of as two exponential distributions spliced together back to back, as well as the two-tailed exponential distribution and the bilateral exponential law (Feller, 1962). Aryal (2006) studied the Laplace and related probability distributions along with their applications to find a probability distribution that could be derived from the Laplace distribution and could be used to frame models for various real-world problems. Nadarajah (2010) obtained two posterior distributions for the mean of the Laplace distribution by deriving the distributions of the product $X Y$ and the ratio $X \mid Y$ when $X$ and $Y$ are Student's $t$ and Laplace random variables distributed independently of each other. Abbasi (2011) considered the Bayesian aspect of a discrete Laplace distribution, and compared the Bayes estimator with that of maximum entropy for the discrete Laplace distribution. Ali, Aslam, Abbas, and Ali Kazmi (2012) estimated the scale 


\section{JAN \& AHMAD}

parameter of the Laplace model using different asymmetric loss functions. The estimates were compared using the posterior risks (PRs) under these loss functions. Rasheed and Emad (2015) obtained Bayesian and non-Bayesian estimates for Laplace distribution under different loss functions and made comparison between them through Monte Carlo simulation, depending on mean square errors (MSEs).

The classical Laplace distribution, also known as first law of Laplace, is a probability distribution on $(-\infty<x<\infty)$ given by the density function

$$
\mathrm{f}(x)=\frac{1}{2 \lambda} \exp \left[-\frac{|x|}{\lambda}\right] \quad \lambda>0,-\infty<x<\infty .
$$

Here, $\lambda$ is a scale parameter. It is a symmetric distribution whose tails fall off less sharply than the Gaussian distribution but faster than the Cauchy distribution. Both the normal and Laplace distributions can be used to analyze symmetric data. It is well-known that the normal distribution is used to analyze symmetric data with short tails, whereas the Laplace model is used for data with long tails. Various forms of the skew Laplace distribution have been introduced and applied in several areas including medical science, environmental science, communications, economics and finance, etc. It can be used to model the difference between the waiting times of two events generated by two random processes. It can also be used to describe breaking strength data, modeling the differences in flood stages, etc. (Krishnamoorthy, 2006).

\section{Normal Approximation}

When the posterior distribution $\mathrm{P}(\lambda \mid x)$ is unimodal and roughly symmetric, the convenient procedure is to approximate it by a normal distribution centered at the mode, yielding the approximation

$$
\mathrm{P}(\lambda \mid x) \sim \mathrm{N}\left\{\hat{\lambda},[\mathrm{I}(\hat{\lambda})]^{-1}\right\} \text {, where } \mathrm{I}(\hat{\lambda})=\frac{-\partial^{2}}{\partial \lambda^{2}} \log \mathrm{P}(\lambda \mid x)
$$

If the mode, $\hat{\lambda}$, is in the interior parameter space, then $I(\lambda)$ is positive; if $\hat{\lambda}$ is a vector parameter, then $\mathrm{I}(\lambda)$ is a matrix.

This was reviewed by Ahmed, Khan, and Ahmad (2007) and Ahmad, Ahmed, and Khan (2011), who discussed the Bayesian analysis of exponential and gamma distribution using normal and Laplace approximations. Further, Sultan and Ahmad 


\section{BAYESIAN ANALYSIS OF LAPLACE DISTRIBUTION}

(2015a, b) obtained the Bayes' estimates of shape parameters of the Topp-Leone and Kumaraswamy distributions under different priors using Bayesian approximation techniques. Moreover, Jan and Ahmad (2016) studied the behavior of shape parameter of the inverse Lomax distribution using Bayesian approximation techniques.

The likelihood function for a random sample $\left(x_{1}, x_{2}, \ldots, x_{n}\right)$, which is taken from the Laplace distribution (1), is given as

$$
\mathrm{L}(x \mid \lambda)=\frac{1}{2^{n} \lambda^{n}} \exp \left[-\frac{\sum_{i=1}^{n}\left|x_{i}\right|}{\lambda}\right]
$$

Using inverted gamma prior

$$
\mathrm{f}(\lambda)=\frac{\beta^{\alpha}}{\Gamma(\alpha)}\left(\frac{1}{\lambda}\right)^{\alpha+1} \exp \left[-\frac{\beta}{\lambda}\right], \quad \alpha, \beta, \lambda>0,
$$

where $\alpha$ and $\beta$ are known hyperparameters, the posterior distribution for $\lambda$ is

$$
\mathrm{P}(\lambda \mid x) \propto\left(\frac{1}{\lambda}\right)^{n+\alpha+1} \exp \left[-\frac{(\beta+T)}{\lambda}\right], \quad \text { where } T=\sum_{i=1}^{n}\left|x_{i}\right|,
$$

which is the density similar to the inverted Gamma distribution.

The log posterior is

$$
\log \mathrm{P}(\lambda \mid x)=-(n+\alpha+1) \log \lambda-\frac{(\beta+T)}{\lambda} .
$$

The first derivative is

$$
\frac{\partial}{\partial \lambda} \log \mathrm{P}(\lambda \mid x)=\frac{-(n+\alpha+1)}{\lambda}+\frac{(\beta+T)}{\lambda^{2}},
$$

and the posterior mode is obtained as 


\section{JAN \& AHMAD}

$$
\hat{\lambda}=\frac{\beta+T}{n+\alpha+1} .
$$

The second-order derivative of the log posterior density is given by

$$
\frac{\partial^{2}}{\partial \lambda^{2}} \log \mathrm{P}(\lambda \mid x)=\frac{(n+\alpha+1)}{\lambda^{2}}-2 \frac{(\beta+T)}{\lambda^{3}} .
$$

Therefore, the negative Hessian is

$$
\begin{aligned}
\mathrm{I}(\hat{\lambda}) & =\frac{-\partial^{2} \log \mathrm{P}(\lambda \mid x)}{\partial \lambda^{2}}=\frac{(n+\alpha+1)}{\lambda^{2}}+\frac{2(\beta+T)}{\lambda^{3}} \\
& \Rightarrow[\mathrm{I}(\hat{\lambda})]^{-1}=\frac{(\beta+T)^{2}}{(n+\alpha+1)^{3}}
\end{aligned}
$$

Thus, the posterior distribution can be approximated as

$$
\mathrm{P}(\lambda \mid x) \sim \mathrm{N}\left[\left(\frac{\beta+T}{n+\alpha+1}\right), \frac{(\beta+T)^{2}}{(n+\alpha+1)^{3}}\right] .
$$

Using an inverted chi-square prior,

$$
f(\lambda)=\frac{\left(\frac{b}{2}\right)^{\frac{a}{2}}}{2^{\frac{a}{2}}}\left(\frac{1}{\lambda}\right)^{\frac{a}{2}+1} \exp \left[-\frac{b}{2 \lambda}\right], \quad a, b, \lambda>0
$$

with hyper parameters $(a, b)$; the posterior distribution of $\lambda$ for the given data $\left(x_{1}, x_{2}, \ldots, x_{n}\right)$ is given by

$$
\mathrm{P}(\lambda \mid x) \propto \frac{1}{\lambda^{\left(\frac{a}{2}+n+1\right)}} \exp \left[-\frac{\left[\frac{b}{2}+T\right]}{\lambda}\right], \quad \lambda>0
$$




\section{BAYESIAN ANALYSIS OF LAPLACE DISTRIBUTION}

The log posterior is

$$
\log \mathrm{P}(\lambda \mid x)=-\left(\frac{a}{2}+n+1\right) \log \lambda-\frac{\left(\frac{b}{2}+T\right)}{\lambda}
$$

The first derivative is

$$
\frac{\partial}{\partial \lambda} \log \mathrm{P}(\lambda \mid x)=\frac{-\left(\frac{a}{2}+n+1\right)}{\lambda}+\frac{\left(\frac{b}{2}+T\right)}{\lambda^{2}},
$$

and the posterior mode is obtained as

$$
\hat{\lambda}=\frac{\left(\frac{b}{2}+T\right)}{\left(\frac{a}{2}+n+1\right)}
$$

The second-order derivative of the log posterior density is given by

$$
\begin{aligned}
\mathrm{I}(\hat{\lambda}) & =\frac{-\partial^{2} \log \mathrm{P}(\lambda \mid x)}{\partial \lambda^{2}}=\frac{\left(n+\frac{a}{2}+1\right)^{3}}{\left(\frac{b}{2}+T\right)^{2}} \\
& \Rightarrow[\mathrm{I}(\hat{\lambda})]^{-1}=\frac{\left(\frac{b}{2}+T\right)^{2}}{\left(n+\frac{a}{2}+1\right)^{3}}
\end{aligned}
$$

Thus, the posterior distribution can be approximated as 


\section{JAN \& AHMAD}

$$
\mathrm{P}(\lambda \mid x) \sim \mathrm{N}\left[\left(\frac{\frac{b}{2}+T}{n+\frac{a}{2}+1}\right), \frac{\left(\frac{b}{2}+T\right)^{2}}{\left(n+\frac{a}{2}+1\right)^{3}}\right] .
$$

Using a Gumbel type II prior, an informative prior with hyperparameter $\gamma$,

$$
\mathrm{f}(\lambda)=\gamma\left(\frac{1}{\lambda}\right)^{2} \exp \left(\frac{-\gamma}{\lambda}\right), \quad \gamma, \lambda>0,
$$

we obtain the posterior distribution of $\lambda$ for the given data $\left(x_{1}, x_{2}, \ldots, x_{n}\right)$ as

$$
\mathrm{P}(\lambda \mid x) \propto \frac{1}{\lambda^{n+2}} \exp \left[-\frac{(\gamma+T)}{\lambda}\right] .
$$

The log posterior is given by

$$
\log \mathrm{P}(\lambda \mid x)=-(2+n) \log \lambda-\frac{(\gamma+T)}{\lambda}
$$

and the posterior mode is obtained as

$$
\hat{\lambda}=\frac{\gamma+T}{n+2} .
$$

The second-order derivative of the log posterior density is given as

$$
\frac{-\partial^{2}}{\partial \lambda^{2}} \log \mathrm{P}(\lambda \mid x)=\frac{n+2}{\lambda^{2}}-\frac{2(\gamma+T)}{\lambda^{3}} .
$$

The negative Hessian is

$$
\mathrm{I}(\hat{\lambda})=\frac{-\partial^{2}}{\partial \lambda^{2}} \log \mathrm{P}(\lambda \mid x)=\frac{(n+2)^{3}}{(\gamma+T)^{2}} .
$$




\section{BAYESIAN ANALYSIS OF LAPLACE DISTRIBUTION}

Therefore,

$$
[\mathrm{I}(\hat{\lambda})]^{-1}=\frac{(\gamma+T)^{2}}{(n+2)^{3}}
$$

Thus, the posterior distribution can be approximated as

$$
\mathrm{P}(\lambda \mid x) \sim \mathrm{N}\left[\left(\frac{\gamma+T}{n+2}\right), \frac{(\gamma+T)^{2}}{(n+2)^{3}}\right]
$$

\section{T-K Approximation}

Lindley's approximation method requires the computation of higher-order partial derivatives, which is usually tedious to calculate when the parameter $\lambda$ is vectorvalued. Tierney and Kadane (1986) gave the Laplace method to evaluate $\mathrm{E}[\mathrm{h}(\lambda) \mid x]$ as

$$
\mathrm{E}[\mathrm{h}(\lambda) \mid x] \cong \frac{\hat{\varphi}^{*} \exp \left[-n \mathrm{~h}^{*}\left(\hat{\lambda}^{*}\right)\right]}{\varphi \exp [-n \mathrm{~h}(\hat{\lambda})]}
$$

where

$$
\begin{aligned}
& -n \mathrm{~h}(\hat{\lambda})=\log \mathrm{P}(\lambda \mid x), \quad-n \mathrm{~h}^{*}\left(\hat{\lambda}^{*}\right)=\log \mathrm{P}(\lambda \mid x)+\log \mathrm{h}(\lambda), \\
& \hat{\varphi}^{2}=-\left[-n \mathrm{~h}^{\prime \prime}(\hat{\lambda})\right]^{-1}, \quad \hat{\varphi}^{* 2}=-\left[-n \mathrm{~h}^{\prime \prime}\left(\hat{\lambda}^{*}\right)\right]^{-1}
\end{aligned}
$$

Thus, for the Laplace model, T-K approximation for the scale parameter $\lambda$ is obtained, under inverted gamma prior, as

$$
\mathrm{f}(\lambda) \propto\left(\frac{1}{\lambda}\right)^{\alpha+1} \exp \left[-\frac{\beta}{\lambda}\right]
$$

the posterior distribution for the scale parameter $\lambda$ is calculated in (4) 


\section{JAN \& AHMAD}

$$
\begin{aligned}
& -n \mathrm{~h}(\lambda)=-(n+\alpha+1) \log \lambda-\frac{(\beta+T)}{\lambda} ; \quad-n \mathrm{~h}^{\prime}(\lambda)=\frac{-(n+\alpha+1)}{\lambda}+\frac{\beta+T}{\lambda^{2}} \\
& \Rightarrow \hat{\lambda}=\frac{\beta+T}{n+\alpha+1}
\end{aligned}
$$

Also,

$$
-n \mathrm{~h}^{\prime \prime}(\hat{\lambda})=-\frac{(n+\alpha+1)^{3}}{(\beta+T)^{2}}
$$

Therefore,

$$
\hat{\varphi}^{2}=\frac{(\beta+T)^{2}}{(n+\alpha+1)^{3}} \quad \text { or } \quad \hat{\varphi}=\frac{\beta+T}{(n+\alpha+1)^{\frac{3}{2}}}
$$

Now,

$$
-n \mathrm{~h}^{*}\left(\lambda^{*}\right)=-n \mathrm{~h}(\lambda)+\log \mathrm{h}(\lambda)=(n+\alpha) \log \lambda^{*}-\frac{(\beta+T)}{\lambda^{*}}
$$

Also,

$$
-n \mathrm{~h}^{* *}\left(\lambda^{*}\right)=\frac{-(n+\alpha)}{\lambda^{*}}+\frac{\beta+T}{\lambda^{* 2}} \Rightarrow \hat{\lambda}^{*}=\frac{\beta+T}{n+\alpha} .
$$

Further,

$$
-n \mathrm{~h}^{\prime \prime}\left(\hat{\lambda}^{*}\right)=\frac{(n+\alpha)}{\lambda^{2}}-\frac{2(\beta+T)}{\lambda^{3}}=-\frac{(n+\alpha)^{3}}{(\beta+T)^{2}} \Rightarrow \hat{\varphi}^{*}=\frac{\beta+T}{(n+\alpha)^{\frac{3}{2}}} .
$$

Using the values in (10), 


\section{BAYESIAN ANALYSIS OF LAPLACE DISTRIBUTION}

$$
\begin{aligned}
\mathrm{E}(\lambda \mid x) & =\left(\frac{n+\alpha+1}{n+\alpha}\right)^{\frac{3}{2}} \frac{\exp \left[-(n+\alpha) \log \hat{\lambda}^{*}-\frac{\beta+T}{\hat{\lambda}^{*}}\right]}{\exp \left[-(n+\alpha+1) \log \hat{\lambda}-\frac{\beta+T}{\hat{\lambda}}\right]} \\
& =\left(\frac{n+\alpha}{n+\alpha+1}\right)^{n+\alpha-\frac{3}{2}}\left(\frac{\beta+T}{n+\alpha+1}\right) e
\end{aligned}
$$

Similarly,

$$
\mathrm{E}\left(\lambda^{2} \mid x\right)=\frac{\hat{\varphi}^{*} \exp \left[-n \mathrm{~h}^{*}\left(\hat{\lambda}^{*}\right)\right]}{\hat{\varphi} \exp [-n \mathrm{~h}(\hat{\lambda})]}
$$

here, $-n \mathrm{~h}^{*}\left(\hat{\lambda}^{*}\right)=\log \lambda^{2}-n \mathrm{~h}(\lambda)$.

$$
\begin{aligned}
-n \mathrm{~h}^{*}\left(\hat{\lambda}^{*}\right) & =-(n+\alpha-1) \log \lambda-\frac{(\beta+T)}{\lambda} \text { and } \\
-n \mathrm{~h}^{\prime *}\left(\hat{\lambda}^{*}\right) & =\frac{-(n+\alpha-1)}{\lambda}+\frac{\beta+T}{\lambda^{2}} \\
& \Rightarrow \hat{\lambda}^{*}=\frac{\beta+T}{n+\alpha-1}
\end{aligned}
$$

Now,

$$
-n \mathrm{~h}^{\prime *}\left(\hat{\lambda}^{*}\right)=\frac{(n+\alpha-1)}{\lambda^{2}}-2 \frac{(\beta+T)}{\lambda^{3}}=-\frac{(n+\alpha-1)^{3}}{(\beta+T)^{2}}
$$

Then,

$$
\hat{\varphi}^{*}=\frac{\beta+T}{(n+\alpha-1)^{\frac{3}{2}}} .
$$

Therefore, 


\section{JAN \& AHMAD}

$$
\begin{aligned}
\mathrm{E}\left[\lambda^{2} \mid x\right] & =\left(\frac{n+\alpha+1}{n+\alpha-1}\right)^{\frac{3}{2}} \frac{\exp \left[-(n+\alpha-1) \log \hat{\lambda}^{*}-\frac{(\beta+T)}{\hat{\lambda}^{*}}\right]}{\exp \left[-(n+\alpha+1) \log \lambda-\frac{(\beta+T)}{\hat{\lambda}}\right]} \\
& =\left(\frac{n+\alpha-1}{n+\alpha+1}\right)^{n+\alpha-\frac{5}{2}}\left(\frac{\beta+T}{n+\alpha+1}\right)^{2} e^{2}
\end{aligned}
$$

Hence,

$$
\begin{aligned}
\text { Variance } & =\mathrm{E}\left[\lambda^{2} \mid x\right]-\{\mathrm{E}[\lambda \mid x]\}^{2} \\
& =\left(\frac{n+\alpha-1}{n+\alpha+1}\right)^{n+\alpha-\frac{5}{2}} \frac{(\beta+T)^{2}}{(n+\alpha+1)^{2}} e^{2}-\left[\left(\frac{n+\alpha}{n+\alpha+1}\right)^{n+\alpha-\frac{3}{2}}\left(\frac{\beta+T}{n+\alpha+1}\right) e\right]^{2}
\end{aligned}
$$

Using an inverted chi square prior,

$$
\mathrm{f}(\lambda) \propto\left(\frac{1}{\lambda}\right)^{\frac{a}{2}+1} \exp \left[\frac{-b}{2 \lambda}\right],
$$

the posterior distribution for $\lambda$ is given by equation (6):

$$
\begin{aligned}
& -n \mathrm{~h}(\lambda)=-\left(n+\frac{a}{2}+1\right) \log \lambda-\frac{\left(\frac{b}{2}+T\right)}{\lambda} ;-n \mathrm{~h}^{\prime}(\lambda)=\frac{-\left(n+\frac{a}{2}+1\right)}{\lambda}+\frac{\frac{b}{2}+T}{\lambda^{2}} \\
& \Rightarrow \hat{\lambda}=\frac{\frac{b}{2}+T}{n+\frac{a}{2}+1}
\end{aligned}
$$

Now, 


\section{BAYESIAN ANALYSIS OF LAPLACE DISTRIBUTION}

$$
-n \mathrm{~h}^{\prime \prime}(\hat{\lambda})=-\frac{-\left(n+\frac{a}{2}+1\right)^{3}}{\left(\frac{b}{2}+T\right)^{2}} \Rightarrow \hat{\varphi}=\frac{\frac{b}{2}+T}{\left(n+\frac{a}{2}+1\right)^{\frac{3}{2}}}
$$

Also,

$$
\begin{aligned}
& -n \mathrm{~h}^{*}\left(\lambda^{*}\right)=-n \mathrm{~h}(\lambda)+\log \mathrm{h}(\lambda)=-\left(n+\frac{a}{2}\right) \log \lambda-\frac{\left(\frac{b}{2}+T\right)}{\lambda} \\
& -n \mathrm{~h}^{\prime *}\left(\lambda^{*}\right)=-\frac{\left(n+\frac{a}{2}\right)}{\lambda}+\frac{\frac{b}{2}+T}{\lambda^{2}} \Rightarrow \hat{\lambda}^{*}=\frac{\frac{b}{2}+T}{n+\frac{a}{2}}
\end{aligned}
$$

Then,

$$
-n \mathrm{~h}^{\prime \prime}\left(\hat{\lambda}^{*}\right)=-\frac{\left(n+\frac{a}{2}\right)^{3}}{\left(\frac{b}{2}+T\right)^{2}} \Rightarrow \hat{\varphi}^{*}=\frac{\frac{b}{2}+T}{\left(n+\frac{a}{2}\right)^{\frac{3}{2}}}
$$

Therefore,

$$
\mathrm{E}[\lambda \mid x]=\frac{\hat{\varphi}^{*} \exp \left[-n \mathrm{~h}^{*}\left(\hat{\lambda}^{*}\right)\right]}{\hat{\varphi} \exp [-n h(\hat{\lambda})]}=\left(\frac{n+\frac{a}{2}}{n+\frac{a}{2}+1}\right)^{n+\frac{a}{2}-\frac{3}{2}}\left(\frac{\frac{b}{2}+T}{n+\frac{a}{2}+1}\right) e .
$$

Now,

$$
\mathrm{E}\left(\lambda^{2} \mid x\right)=\frac{\hat{\varphi}^{*} \exp \left[-n \mathrm{~h}^{*}\left(\hat{\lambda}^{*}\right)\right]}{\hat{\varphi} \exp [-n h(\hat{\lambda})]}
$$




\section{JAN \& AHMAD}

Here $-n \mathrm{~h}^{*}\left(\hat{\lambda}^{*}\right)=\log \lambda^{2}-n \mathrm{~h}(\lambda)$,

$$
\begin{gathered}
\Rightarrow \mathrm{E}\left[\lambda^{2} \mid x\right]=\left(\frac{n+\frac{a}{2}-1}{n+\frac{a}{2}+1}\right)^{n+\frac{a}{2}-\frac{5}{2}}\left(\frac{\frac{b}{2}+T}{n+\frac{a}{2}+1}\right) e^{2}, \\
\mathrm{~V}[\lambda \mid x]=\left(\frac{n+\frac{a}{2}-1}{n+\frac{a}{2}+1}\right)^{n+\frac{a}{2}-\frac{5}{2}}\left(\frac{\frac{b}{2}+T}{n+\frac{a}{2}+1}\right)^{2} e^{2}-\left\{\left(\frac{n+\frac{a}{2}}{n+\frac{a}{2}+1}\right)^{n+\frac{a}{2}-\frac{3}{2}}\left(\frac{\frac{b}{2}+T}{n+\frac{a}{2}+1}\right) e\right\}^{2}
\end{gathered}
$$

Using a Gumbel type II prior,

$$
\mathrm{f}(\lambda) \propto\left(\frac{1}{\lambda}\right)^{2} \exp \left(-\frac{\gamma}{\lambda}\right), \quad \gamma, \lambda>0,
$$

the posterior distribution for the parameter $\lambda$ is given by (9):

$$
-n \mathrm{~h}(\lambda)=\log \mathrm{P}(\lambda \mid x)=-(n+2) \log \lambda-\frac{(\gamma+T)}{\lambda},
$$

and

$$
-n \mathrm{~h}^{\prime}(\lambda)=\frac{-(n+2)}{\lambda}+\frac{(\gamma+T)}{\lambda^{2}} \Rightarrow \hat{\lambda}=\frac{\gamma+T}{n+2}
$$

Also,

$$
-n \mathrm{~h}^{\prime \prime}(\hat{\lambda})=-\frac{-(n+2)^{3}}{(\gamma+T)^{2}} \Rightarrow \hat{\varphi}=\frac{\gamma+T}{(n+2)^{\frac{3}{2}}} .
$$

Now, 


\section{BAYESIAN ANALYSIS OF LAPLACE DISTRIBUTION}

$$
-n \mathrm{~h}^{*}\left(\lambda^{*}\right)=-n \mathrm{~h}(\lambda)+\log \mathrm{h}(\lambda)=-(n+1) \log \lambda-\frac{(\gamma+T)}{\lambda}
$$

Then,

$$
-n \mathrm{~h}^{* *}\left(\lambda^{*}\right)=-\frac{-(n+1)}{\lambda}+\frac{(\gamma+T)}{\lambda^{2}} \Rightarrow \hat{\lambda}^{*}=\frac{\gamma+T}{n+1}
$$

Further,

$$
-n \mathrm{~h}^{\prime \prime}\left(\hat{\lambda}^{*}\right)=-\frac{-(n+1)^{3}}{(\gamma+T)^{2}} \Rightarrow \hat{\varphi}^{*}=\frac{\gamma+T}{(n+1)^{\frac{3}{2}}} .
$$

Therefore,

$$
\begin{aligned}
\mathrm{E}(\lambda \mid x) & =\frac{\hat{\varphi}^{*} \exp \left[-n \mathrm{~h}^{*}\left(\hat{\lambda}^{*}\right)\right]}{\hat{\varphi} \exp [-n h(\hat{\lambda})]} \\
& =\left(\frac{n+1}{n+2}\right)^{n-\frac{1}{2}}\left(\frac{\gamma+T}{n+2}\right) e
\end{aligned}
$$

Now,

$$
\mathrm{E}\left(\lambda^{2} \mid x\right)=\frac{\hat{\varphi}^{*} \exp \left[-n \mathrm{~h}^{*}\left(\lambda^{*}\right)\right]}{\hat{\varphi} \exp [-n \mathrm{~h}(\lambda)]}
$$

where $-n \mathrm{~h}^{*}\left(\lambda^{*}\right)=\log \lambda^{2}-n \mathrm{~h}(\lambda)$.

$$
\begin{gathered}
\Rightarrow \mathrm{E}\left(\lambda^{2} \mid x\right)=\left(\frac{n}{n+2}\right)^{n-\frac{3}{2}}\left(\frac{\gamma+T}{n+2}\right)^{2} e^{2} \\
\therefore \mathrm{V}[\lambda \mid x]=\left(\frac{n}{n+2}\right)^{n-\frac{3}{2}}\left(\frac{\gamma+T}{n+2}\right)^{2} e^{2}-\left[\left(\frac{n+1}{n+2}\right)^{n-\frac{1}{2}}\left(\frac{\gamma+T}{n+2}\right) e\right]^{2}
\end{gathered}
$$


JAN \& AHMAD

Table 1. Posterior estimates of Laplace distribution using normal approximation

\begin{tabular}{|c|c|c|c|c|c|c|c|c|c|c|}
\hline \multirow[b]{2}{*}{$n$} & \multirow[b]{2}{*}{$\lambda$} & \multicolumn{3}{|c|}{ Inverse gamma prior } & \multicolumn{3}{|c|}{ Inverse chi square prior } & \multicolumn{3}{|c|}{ Gumbel type II prior } \\
\hline & & $\alpha=\beta=0.5$ & $\alpha=\beta=1$ & $\alpha=\beta=2$ & $a=b=0.5$ & $a=b=1$ & $a=b=2$ & $y=0.5$ & $y=1$ & $y=2$ \\
\hline \multirow[t]{6}{*}{25} & 0.5 & 0.63052 & 0.63736 & 0.65031 & 0.62700 & 0.63052 & 0.63736 & 0.61884 & 0.63736 & 0.67440 \\
\hline & & $(0.01499)$ & $(0.01503)$ & $(0.01509)$ & $(0.01496)$ & $(0.01499)$ & $(0.01503)$ & $(0.01417)$ & $(0.01503)$ & $(0.01683)$ \\
\hline & 1.0 & 0.88203 & 0.88422 & 0.88835 & 0.88091 & 0.88203 & 0.88422 & 0.86570 & 0.92126 & 0.92126 \\
\hline & & $(0.02934)$ & $(0.02895)$ & $(0.02817)$ & $(0.02955)$ & $(0.02934)$ & $(0.02894)$ & (0.02774) & $(0.02894)$ & $(0.03142)$ \\
\hline & 2.0 & 2.22916 & 2.20640 & 2.16332 & 2.24087 & 2.22916 & 2.20640 & 2.18788 & 2.20640 & 2.24344 \\
\hline & & $(0.18750)$ & $(0.18029)$ & $(0.16713)$ & $(0.19128)$ & $(0.18750)$ & $(0.18029)$ & $(0.03250)$ & $(0.18029)$ & $(0.18639)$ \\
\hline \multirow[t]{6}{*}{50} & 0.5 & 0.49012 & 0.49502 & 0.50455 & 0.48763 & 0.49012 & 0.49502 & 0.48541 & 0.49502 & 0.51425 \\
\hline & & $(0.00465)$ & $(0.00470)$ & $(0.00479)$ & $(0.00462)$ & $(0.00465)$ & $(0.00470)$ & $(0.00452)$ & $(0.00470)$ & $(0.00507)$ \\
\hline & 1.0 & 1.09274 & 1.09185 & 1.09011 & 1.09319 & 1.09274 & 1.09185 & 1.08223 & 1.09185 & 1.11108 \\
\hline & & $(0.02317)$ & $(0.02291)$ & $(0.02241)$ & $(0.02330)$ & $(0.02317)$ & $(0.02291)$ & $(0.02251)$ & $(0.02291)$ & $(0.02373)$ \\
\hline & 2.0 & 2.32566 & 2.31292 & 2.28814 & 2.33213 & 2.32566 & 2.31292 & 2.30330 & 2.31292 & 2.33215 \\
\hline & & $(0.10501)$ & $(0.10286)$ & $(0.09877)$ & $(0.10611)$ & $(0.10501)$ & $(0.10286)$ & $(0.10201)$ & $(0.10286)$ & $(0.10458)$ \\
\hline \multirow[t]{6}{*}{100} & 0.5 & 0.45510 & 0.45777 & 0.46304 & 0.45376 & 0.45510 & 0.45777 & 0.45287 & 0.45777 & 0.46758 \\
\hline & & $(0.0$ & $(0.00204)$ & $(0.0$ & $(0.00202)$ & $(0.00$ & $(0.00$ & $(0.00200)$ & $(0.00204)$ & $(0.00213)$ \\
\hline & 1.0 & 1.13627 & 1.13560 & 1.13428 & 1.13660 & 1.13627 & 1.13560 & 1.13070 & 1.13560 & 1.14540 \\
\hline & & $(0.01271)$ & $(0.01263)$ & $(0.01248)$ & $(0.01274)$ & $(0.01271)$ & $(0.01263)$ & $(0.01252)$ & $(0.01263)$ & $(0.01285)$ \\
\hline & 2.0 & 2.21633 & 2.21036 & 2.19861 & 2.21933 & 2.21633 & 2.21036 & 2.20546 & 2.21036 & 2.22017 \\
\hline & & $(0.04838)$ & $(0.04838)$ & $(0.04692)$ & $(0.04863)$ & $(0.04838)$ & $(0.04788)$ & $(0.04767)$ & $(0.04788)$ & $(0.04831)$ \\
\hline
\end{tabular}




\section{BAYESIAN ANALYSIS OF LAPLACE DISTRIBUTION}

Table 2. Posterior estimates of Laplace distribution using T-K approximation

\begin{tabular}{|c|c|c|c|c|c|c|c|c|c|c|}
\hline \multirow[b]{2}{*}{$n$} & \multirow[b]{2}{*}{$\lambda$} & \multicolumn{3}{|c|}{ Inverse gamma prior } & \multicolumn{3}{|c|}{ Inverse chi square prior } & \multicolumn{3}{|c|}{ Gumbel type II prior } \\
\hline & & $\alpha=\beta=0.5$ & $\alpha=\beta=1$ & $\alpha=\beta=2$ & $a=b=0.5$ & $a=b=1$ & $a=b=2$ & $y=0.5$ & $y=1$ & $y=2$ \\
\hline \multirow[t]{6}{*}{25} & 0.5 & 0.35256 & 0.36549 & 0.38988 & 0.34589 & 0.35256 & 0.36549 & 0.34553 & 0.36549 & 0.40543 \\
\hline & & $(0.00526)$ & $(0.00553)$ & $(0.00605)$ & $(0.00511)$ & $(0.00526)$ & $(0.00553)$ & $(0.00494)$ & $(0.00553)$ & $(0.00681)$ \\
\hline & 1.0 & 1.39540 & 1.38754 & 1.37273 & 1.39944 & 1.39540 & 1.38754 & 1.36757 & 1.38754 & 1.42748 \\
\hline & & $(0.08257)$ & $(0.07994)$ & $(0.07513)$ & $(0.08393)$ & $(0.08257)$ & $(0.07994)$ & $(0.07766)$ & $(0.07994)$ & $(0.08461)$ \\
\hline & 2.0 & 2.32739 & 2.30095 & 2.25111 & 2.34101 & 2.32739 & 2.30095 & 2.28098 & 2.30095 & 2.34089 \\
\hline & & $(0.22970)$ & $(0.21987)$ & $(0.20207)$ & $(0.23488)$ & $(0.22970)$ & $(0.21987)$ & $(0.21607)$ & $(0.21987)$ & $(0.22756)$ \\
\hline \multirow[t]{6}{*}{50} & 0.5 & 0.56176 & 0.56614 & 0.57465 & 0.55954 & 0.56176 & 0.56614 & 0.55615 & 0.56614 & 0.58613 \\
\hline & & $(0.00649)$ & $(0.00652)$ & $(0.00658)$ & $(0.00647)$ & $(0.00649)$ & $(0.00652)$ & $(0.00629)$ & $(0.00652)$ & $(0.00699)$ \\
\hline & 1.0 & 1.11816 & 1.11699 & 1.11470 & 1.11876 & 1.11816 & 1.11699 & 1.10699 & 1.11699 & 1.13698 \\
\hline & & $(0.02574)$ & $(0.02543)$ & $(0.02482)$ & $(0.02590)$ & $(0.02574)$ & $(0.02543)$ & $(0.02497)$ & $(0.02543)$ & $(0.02635)$ \\
\hline & 2.0 & 1.76988 & 1.76219 & 1.74726 & 1.77378 & 1.76988 & 1.76219 & 1.75219 & 1.76219 & 1.78218 \\
\hline & & $(0.06452)$ & $(0.06332)$ & (0.06099) & $(0.06514)$ & $(0.06452)$ & $(0.06331)$ & $(0.06259)$ & $(0.06331)$ & $(0.06475)$ \\
\hline \multirow[t]{6}{*}{100} & 0.5 & 0.49407 & 0.49660 & 0.50158 & 0.49280 & 0.49407 & 0.49660 & 0.49160 & 0.49660 & 0.50660 \\
\hline & & $(0.00246)$ & $(0.00248)$ & $(0.00250)$ & $(0.00246)$ & $(0.00246)$ & $(0.00248)$ & $(0.00243)$ & $(0.00248)$ & $(0.00258)$ \\
\hline & 1.0 & 1.03193 & 1.03177 & 1.03145 & 1.03201 & 1.03193 & 1.03177 & 1.02677 & 1.03177 & 1.04177 \\
\hline & & $(0.01079)$ & $(0.01074)$ & $(0.01062)$ & $(0.01082)$ & (0.01079) & $(0.01074)$ & $(0.01063)$ & $(0.01074)$ & $(0.01096)$ \\
\hline & 2.0 & 1.90119 & 1.89668 & 1.88781 & 1.90345 & 1.90119 & 1.89668 & 1.89168 & 1.89668 & 1.90668 \\
\hline & & $(0.03667)$ & $(0.03631)$ & $(0.03631)$ & $(0.03685)$ & $(0.03667)$ & $(0.03631)$ & $(0.03612)$ & $(0.03631)$ & $(0.03670)$ \\
\hline
\end{tabular}


JAN \& AHMAD

Table 3. Posterior estimates of Laplace distribution using normal approximation

\begin{tabular}{|c|c|c|c|c|c|c|c|c|c|}
\hline & \multicolumn{3}{|c|}{ Inverse gamma prior } & \multicolumn{3}{|c|}{ Inverse chi square prior } & \multicolumn{3}{|c|}{ Gumbel type II prior } \\
\hline & $\alpha=\beta=0.5$ & $\alpha=\beta=1$ & $\alpha=\beta=2$ & $a=b=0.5$ & $a=b=1$ & $a=b=2$ & $y=0.5$ & $y=1$ & $y=2$ \\
\hline Posterior mean & 84.05195 & 82.98718 & 80.93750 & 84.59477 & 84.05195 & 82.98718 & 82.97436 & 82.98718 & 83.01282 \\
\hline Posterior variance & 183.4995 & 176.5865 & 163.7720 & 187.0922 & 183.4995 & 176.5865 & 176.5319 & 176.5865 & 176.6956 \\
\hline
\end{tabular}

Table 4. Posterior estimates of Laplace distribution using T-K approximation

\begin{tabular}{|c|c|c|c|c|c|c|c|c|c|}
\hline & \multicolumn{3}{|c|}{ Inverse gamma prior } & \multicolumn{3}{|c|}{ Inverse chi square prior } & \multicolumn{3}{|c|}{ Gumbel type II prior } \\
\hline & $\alpha=\beta=0.5$ & $\alpha=\beta=1$ & $\alpha=\beta=2$ & $a=b=0.5$ & $a=b=1$ & $a=b=2$ & $y=0.5$ & $y=1$ & $y=2$ \\
\hline Posterior mean & 88.58938 & 87.40748 & 85.13681 & 89.19252 & 88.58938 & 87.40748 & 87.39398 & 87.40748 & 87.43449 \\
\hline Posterior variance & 220.7281 & 211.9021 & 195.6173 & 225.3260 & 220.7281 & 211.9021 & 211.8367 & 211.9021 & 212.0331 \\
\hline
\end{tabular}




\section{BAYESIAN ANALYSIS OF LAPLACE DISTRIBUTION}

\section{Simulation Study}

In a simulation study, samples were generated of sizes $n=25,50$, and 100 to examine the effect of small, medium, and large samples of posterior estimates using $\mathrm{R}$. The performance of Bayes estimates of the scale parameter of the Laplace distribution were compared under different informative priors using the two approximation techniques. The value of the scale parameter $\lambda$ has been chosen as $0.5,1$, and 2 , and the value of the hyperparameters have also been taken as $0.5,1$, and 2. These results have been replicated 5000 times and the estimates have been obtained. The results are presented in the tables below with the posterior variances enclosed in the parentheses.

\section{Results}

From Tables 1 and 2, it is clearly evident that the posterior variance of the scale parameter of the Laplace distribution in most of the cases is minimum under inverse gamma prior, especially when the value of the hyperparameters $\alpha$ and $\beta$ is taken to be 2 .

\section{Real-Life Example}

The data below were discussed by Schmee and Nelson (1977) and show the number, in thousands of miles, at which different locomotive controls failed in a life test involving 96 controls. The test was terminated after 135,000 miles by which time 37 failures had occurred. The failure times for the 37 failed units are:

$22.5,37.5,46.0,48.5,51.5,53.0,54.5,57.5,66.5,68.0,69.5,76.5,77.0$, $78.5,80.0,81.5,82.0,83.0,84.0,91.5,93.5,102.5,107.0,108.5,112.5$, $113.5,116.0,117.0,118.5,119.0,120.0,122.5,123.0,127.5,131.0$, 132.5, 134.0. (p. 3)

\section{Conclusion}

The aim was to focus on the efficiency of the various informative priors used for the scale parameter $\lambda$ of the Laplace distribution under the two Bayesian approximation techniques. It is observed from the simulation study presented in Tables 1 and 2 that, among all the informative priors, the inverse gamma prior proves to be more efficient with minimum posterior variance when the value of 


\section{JAN \& AHMAD}

hyperparameters is taken to be 2 . Further, the results are validated by considering the estimates obtained from a real-life data set as shown in Tables 3 and 4, thereby justifying the results of our simulation study. In addition to this, it can be seen that the values of posterior variance in Tables 1 and 2 decreases with the increase in sample size. Also, it can be seen that normal approximation gives lesser values of posterior variance of shape parameter of the inverse Lomax distribution than T-K approximation in both the generated and real-life data sets.

\section{References}

Abbasi, N. (2011). Comparison of Bayes estimator and maximum entropy estimator for discrete Laplace distribution. International Journal of Contemporary Mathematical Sciences, 6(9), 447-452. Retrieved from http://www.m-hikari.com/ijcms-2011/9-12-2011/abbasiIJCMS9-12-2011.pdf

Abramowitz, M., \& Stegun, I. (1972). Handbook of mathematical functions: With formulas, graphs, and mathematical tables. New York, NY: Dover Publications.

Ahmad, S. P., Ahmed, A., \& Khan, A. A. (2011). Bayesian analysis of gamma distribution using S-PLUS and R software. Asian Journal of Mathematics and Statistics, 4(4), 224-233. doi: 10.3923/ajms.2011.224.233

Ahmed, A. A., Khan, A. A., \& Ahmad, S. P. (2007). Bayesian analysis of exponential distribution in S-PLUS and R software. Sri Lankan Journal of Applied Statistics, 8, 95-109.

Ali, S., Aslam, M., Abbas, N., \& Ali Kazmi, S. M. (2012). Scale parameter estimation of the Laplace model using different asymmetric loss functions. International Journal of Statistics and Probability, 1(1), 105-127. doi: 10.5539/ijsp.v1n1p105

Aryal, G. R. (2006). Study of Laplace and related probability distributions and their applications (Unpublished doctoral thesis). University of South Florida, Tampa, FL. Retrieved from https://scholarcommons.usf.edu/etd/2443/

Feller, W. (1971). An introduction to probability theory and its applications (2nd ed.). New York, NY: Wiley.

Jan, U., \& Ahmad, S. P. (2016). Bayesian analysis of inverse Lomax distribution using approximation techniques. Mathematical Theory and Modeling, 7(7), 1-12. Retrieved from https://iiste.org/Journals/index.php/MTM/article/view/37699 


\section{BAYESIAN ANALYSIS OF LAPLACE DISTRIBUTION}

Krishnamoorthy, K. (2006). Handbook of statistical distributions with applications. New York, NY: Chapman and Hall/CRC. doi:

10.1201/9781420011371

Nadarajah, S. (2010). Some posterior distributions for the Laplace mean.

Acta Mathematica Scientia, 30(1), 330-340. doi: 10.1016/S0252-9602(10)600495

Rasheed, H. A., \& Emad, F. A.-S. (2015). Bayesian and non-Bayesian estimation for the scale parameter of Laplace distribution. Advances in Environmental Biology, 9(14), 226-232.

Schmee, J., \& Nelson, W. (1977). Estimates and approximate confidence limits for (log) normal life distributions from singly censored samples by maximum likelihood (Technical Information Series report no. 76CRD250). Schenectady, NY: General Electric Co., Corporate Research and Development.

Sultan. H., \& Ahmad, S. P. (2015a). Bayesian approximation techniques for Kumaraswamy distribution. Mathematical Theory and Modeling, 5(5), 49-60. Retrieved from https://iiste.org/Journals/index.php/MTM/article/view/22424

Sultan, H., \& Ahmad, S. P. (2015b). Bayesian approximation techniques of Topp-Leone distribution. International Journal of Statistics and Mathematics, 2(2), 66-72. Retrieved from

https://www.premierpublishers.org/ijsm/030520156844

Tierney, L., \& Kadane, J. (1986). Accurate approximations for posterior moments and marginal densities. Journal of the American Statistical Association, 81(393), 82-86. doi: 10.1080/01621459.1986.10478240 